\title{
Compliance with the 2014 ESC/ESA guidelines on non-cardiac surgery preoperative cardiovascular assessment by 5 tertiary Greek hospitals: A retrospective observational study.
}

\author{
G. Kotsovolis ${ }^{1}$, E. Hatzopoulos ${ }^{1}$, A. Trikoupi² , D. Vasilakos ${ }^{3}$, E. Goutziomitrou ${ }^{4}$, C. Bratzou ${ }^{5}$ \\ 1 Department of Anesthesia, 424 Army General Hospital - Thessaloniki (Greece) \\ 2 Department of Anesthesia, Papanikolaou Hospital - Thessaloniki (Greece) \\ 3 Department of Anesthesia and Intensive Care, AHEPA University Hospital - Thessaloniki (Greece) \\ 4 Department of Anesthesia, Papageorgiou Hospital - Thessaloniki (Greece) \\ 5 Department of Anesthesia, Hippokratio Hospital - Thessaloniki (Greece)
}

Background and Goal of Study: In 2014 the European Society of Cardiology and the European Society of Anaesthesiology published guidelines on preoperative assessment and management of non-cardiac surgery patients. The objective of the study was to evaluate the rate of compliance with the task force recommendations by 5 major tertiary Greek hospitals.

Materials and Methods: A retrospective observational cohort study was designed. 5 tertiary Greek hospitals participated in the study between February 2016 and April 2016. The adult ASA 1-4 patients who underwent non-cardiac surgery were included. Patients treated in Intensive Care Unit were excluded. The preanesthetic charts were reviewed and the information about the age, surgical risk, ASA classification, the cardiological history and medication, the physical examination findings, and the tests ordered were recorded. A total of 5009 records were reviewed. The main outcomes were the rates of compliance with the published recommendations of the following parameters: preoperative testing (electrocardiogram [ECG], heart ultrasound, heart imaging stress testing, invasive coronary angiography), continuation or not of medication (b-blockers, statins, angiotensin-converting enzyme inhibitors [ACEIs] and angiotensin-receptor blockers [ARBs]) and overall compliance with the recommendations.

Results and Discussion: 63,8\% (3197) of the preoperative ECGs, $83,8 \%$ (522) of the heart ultrasonographies and $93,2 \%$ (55) of the non-invasive stress tests shouldn't had been performed.
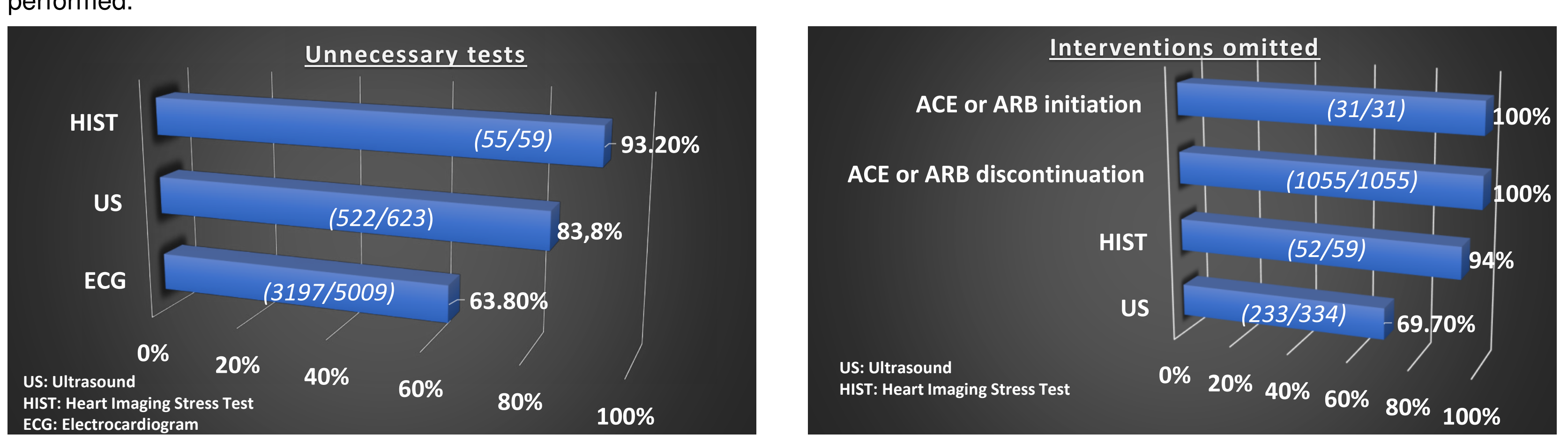

Kristensen SD, Knuuti J, Saraste A et al. 2014 ESC/ESA Guidelines on noncardiac surgery: cardiovascular assessment and management: The Joint Task Force on non-cardiac surgery: cardiovascular assessment and management of the European Society of Cardiology (ESC) and the European Society of Anaesth. Eur J Anaesthesiol 2014; 31: 517-573.
Conclusion(s): The 2014 guidelines for preoperative management of the non-cardiac surgery patients are not sufficiently being followed by the Greek hospitals

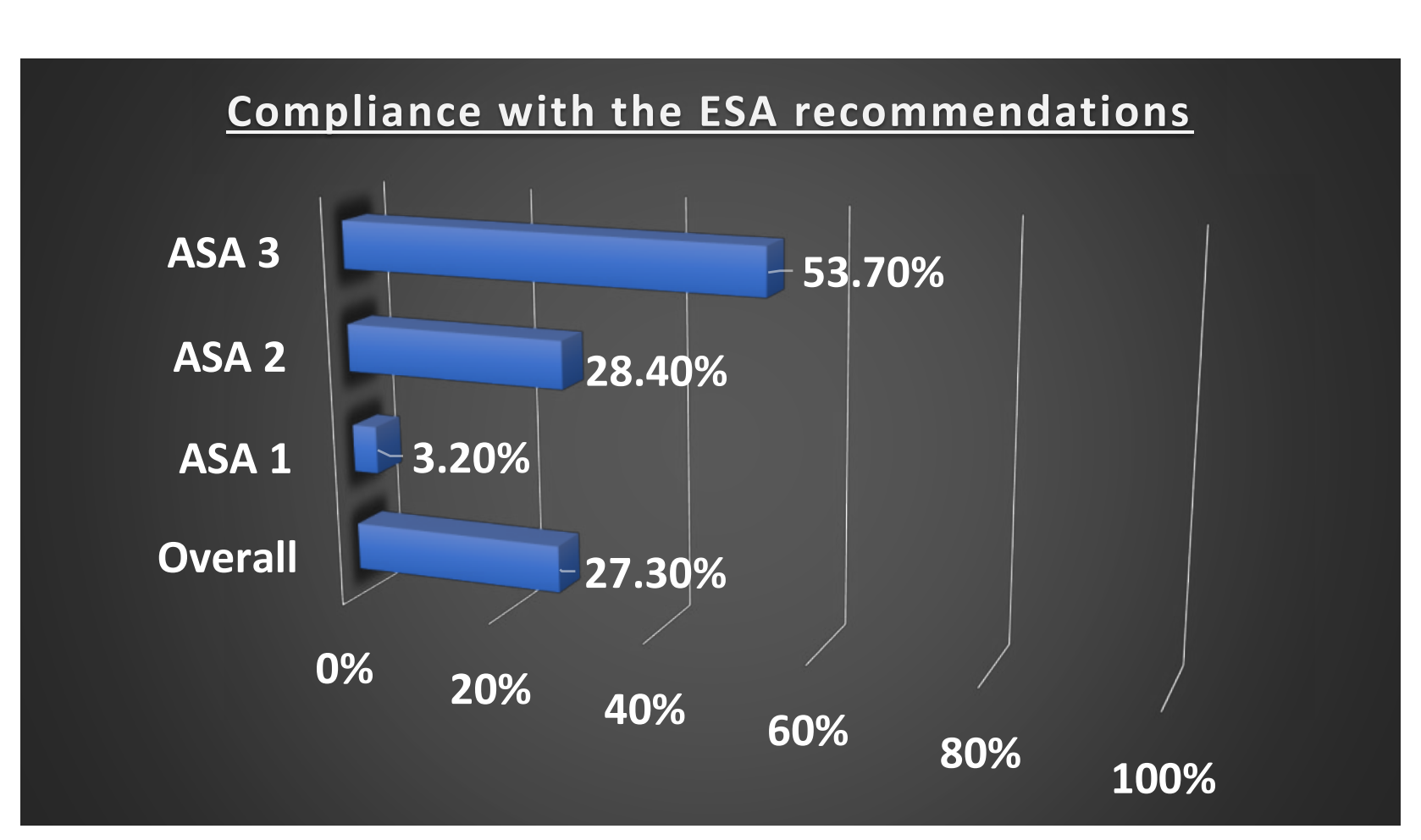

On the other hand, only $30,3 \%(101)$ and $6 \%(4)$ of the patients that should or could have been evaluated with a heart ultrasound and a non-invasive stress test respectively, did the recommended tests. B-blockers and statins were correctly continued to all the patients who were taking them. 1055 patients were receiving ACEls or ARBs for hypertension and should have discontinued their medication, but none did. 53,4\% (31) of the patients with known systolic heart failure were not being treated with ACEls or ARBs as they should and were operated despite the recommendation to begin treatment for at least 1 week before surgery. The overall percentage of the patients managed exactly as recommended was only $27,3 \%$.

Copyright @ 2017 Georgios Kotsovolis, gskotsos@gmail.com 